\title{
Encapsulation of Rhodamine 6G Dye Molecules for Affecting Symmetry of Supramolecular Crystals of Melamine-Barbiturate
}

\author{
Pavel V. Nesterov ${ }^{1}{ }^{1}$, Vladimir V. Shilovskikh ${ }^{1}$, Alexander D. Sokolov ${ }^{1}$, Vladislav V. Gurzhiy ${ }^{2}{ }^{\mathbb{D}}$, \\ Alexander S. Novikov ${ }^{1} \mathbb{D}$, Alexandra A. Timralieva ${ }^{1}$, Elena V. Belogub ${ }^{3}$, Nikolay D. Kondratyuk ${ }^{4,5}{ }^{\oplus}$, \\ Nikita D. Orekhov ${ }^{4,5,6}$ and Ekaterina V. Skorb ${ }^{1, *}$
}

Citation: Nesterov, P.V.; Shilovskikh, V.V.; Sokolov, A.D.; Gurzhiy, V.V.; Novikov, A.S.; Timralieva, A.A.; Belogub, E.V.; Kondratyuk, N.D.; Orekhov, N.D.; Skorb, E.V.

Encapsulation of Rhodamine 6G Dye Molecules for Affecting Symmetry of Supramolecular Crystals of Melamine-Barbiturate. Symmetry 2021, 13, 1119. https://doi.org/ 10.3390/sym13071119

Academic Editor: Victor Borovkov

Received: 17 May 2021

Accepted: 10 June 2021

Published: 23 June 2021

Publisher's Note: MDPI stays neutral with regard to jurisdictional claims in published maps and institutional affiliations.

Copyright: (c) 2021 by the authors. Licensee MDPI, Basel, Switzerland. This article is an open access article distributed under the terms and conditions of the Creative Commons Attribution (CC BY) license (https:// creativecommons.org/licenses/by/ $4.0 /)$.
1 Infochemistry Scientific Center, ITMO University, Lomonosova Str. 9, 191002 Saint-Petersburg, Russia; nesterov@scamt-itmo.ru (P.V.N.); vvshlvskh@itmo.ru (V.V.S.); a.sokolova@infochemistry.ru (A.D.S.); novikov@itmo.ru (A.S.N.); timralieva@infochemistry.ru (A.A.T.)

2 Crystallography Department, Institute of Earth Sciences, Saint-Petersburg State University, University emb. 7/9, 199034 Saint-Petersburg, Russia; vladislav.gurzhiy@spbu.ru

3 South Urals Federal Research Center of Mineralogy and Geoecology, Urals Branch of Russian Academy of Science, 456317 Miass, Russia; belogub@mineralogy.ru

4 Joint Institute for High Temperatures of the Russian Academy of Sciences, 13 Izhorskaya Bd 2, 125412 Moscow, Russia; kondratyuk@phystech.edu (N.D.K.); nikita.orekhov@phystech.edu (N.D.O.)

5 Moscow Institute of Physics and Technology, 9 Institutskiy per., Dolgoprudny, 141701 Moscow, Russia

6 Department of Physics, Bauman Moscow State Technical University, FN-4, 2nd Baumanskaya Str. 5 , 105005 Moscow, Russia

* Correspondence: skorb@itmo.ru

Abstract: Supramolecular organic systems can be used as a host for the encapsulation of small organic molecules. Here, we chose melamine barbiturate as a robust system capable of supramolecular assembly and the Rhodamine 6G dye entrapment as a guest molecule. The encapsulation of the dye was investigated by UV-visible spectroscopy, SEM and optical fluorescent microscopy while the insight into the crystal structure of the system was obtained by single crystal and powder XRD. For investigation of the system's properties on a molecular level, the DFT and Classical Molecular Dynamics methods were utilized. Surprisingly, both theoretical and experimental data show not only the successful encapsulation of Rhodamine 6G molecules inside the supramolecular assembly, but also that inclusion of such molecules leads to the drastic improvement in the organic crystal shape. The melamine barbiturate in presence of the Rhodamine 6G molecules tend to form crystals with lesser degree of twinning and higher symmetry in shape than the ones without dye molecules.

Keywords: encapsulation; supramolecular assembly; organic crystal; DFT; molecular dynamics; crystal structure

\section{Introduction}

The growth in life expectancy and quality of life in recent years has led to the emergence of new approaches in medicine. Recent advances in healthcare stand on the principle of treatment of a target organ or even cell with minimal impact on neighboring tissue. Such an approach set an ambitious task on material science to produce highly functional and at the same time biocompatible materials for a directed and controlled delivery and release of a drug molecule. Such materials must have a number of characteristics: biocompatibility of both the material itself and its components and metabolites, sufficient resistance to external factors and functional transition under gentle conditions [1,2].

Small organic molecules in general and triarylmethane dyes (such as fluorescein) can be used as drugs-like substances for investigating the system for drug encapsulation [3]. Dye molecules are widely used as target objects for encapsulation into various supramolecular structures-whether for protecting them from aggregation or photochemical degradation [4] or for investigating the host-guest chemistry and encapsulating properties of 
various supramolecular systems [5]. Also, the inclusion of dyes into crystalline lattices of organic substances have been reported for the improving properties of photonic crystals $[6,7]$. Fine structure-based tuning of photonic properties of organic dyes is shown for 1D zeolites [8]. Moreover, it was shown that the Rhodamine 6G dye molecule (Figure 1a) was used as a guest molecule for investigating the energy transfer inside different supramolecular hosts $[9,10]$. It was reported that the presence of polyelectrolytes and stirring conditions during crystallization together positively affect the shape of resulting crystals [11]. While polyelectrolytes effectively salt out crystallizing substances, local stirring promotes the growth of crystals with larger linear size.

a)<smiles>CCN=c1cc2oc3cc(NCC)c(C)cc3c(-c3ccccc3C(=O)OCC)c-2cc1C</smiles>

b)<smiles>Nc1nc(N)nc(N)n1</smiles>

c)<smiles>O=C1CC(=O)NC(=O)N1</smiles>

Figure 1. Structural formulas of (a) Rhodamine 6G, (b) Melamine and (c) Barbituric acid.

The systems for encapsulation and drug delivery vary in their mechanism. Some utilized the core-shell structures and some that allow the encapsulation in the structure with the specific adsorption, such as $\pi$-systems [12]. The growth of nonorganic crystals is related to the thermodynamic equilibrium which can be shifted by the inclusion of various substances. Such a mechanism is good for controlling the shape of the crystals but can completely halt the growth [13]. The cooperative growth of the supramolecular assemblies with the formation of the $\pi$-planes for the adsorption is a very prospective field of study since such methods allow the usage of a wide variety of substances and do not require their initial assembly into the standalone aggregates.

It was shown previously in our work that melamine barbiturates (Figure $1 \mathrm{~b}, \mathrm{c}$ ) are capable of forming the highly ordered supramolecular assemblies [14] that can encapsulate some amount of small organic molecules (such as dyes). The in-depth analysis of a very similar supramolecular assembly of melamine cyanurate has shown that stabilization of such self-assembly occurs predominantly via $\pi-\pi$ stacking [15]. Melamine barbiturates (M-BA) exhibit several remarkable properties, such as structure-dependent radical trap activity or inhomogeneous luminescence $[14,16]$. The M-BA structures are considered as candidates for drug delivery systems: the systems based on barbiturate derivatives were proposed as drug-delivery systems [17]. In addition, the similar system, melamine cyanurate, was reported to be promising as a molecule guest box [18]. In its simplest expression, the supramolecular assembly is two or more molecules that join together by noncovalent bonds to form a supramolecule. The range of possible supramolecular structures depends on the strength of interaction between molecules, stable structures with 
covalent-like bonds as well as quasi-stable assemblies that may be obtained. Therefore, we decided to use a melamine barbiturate supramolecular system as a host for encapsulating triarylmethane dye Rhodamine 6G (Rh6G).

Consequently, this study aims to investigate the processes of encapsulation of Rh6G dye in the supramolecular structures of melamine barbiturate: at the early stages of nucleation and during crystal growth in aqueous solutions. Therefore, the relevance of this work also lies in the creation of systems capable of encapsulating drugs and their use in the targeted delivery of drugs. Moreover, our study aims to fill the existing knowledge gap in the understanding of the early stages of growth of binary self-assembly materials. This may enable controlled nanoengineering of materials with desired properties.

\section{Materials and Methods}

Reagents. Melamine (M) $\left(\mathrm{C}_{3} \mathrm{H}_{6} \mathrm{~N}_{6}, 99.0 \%\right.$, Sigma Aldrich, St. Louis, MI, USA), barbituric acid (BA) $\left(\mathrm{C}_{4} \mathrm{H}_{4} \mathrm{~N}_{2} \mathrm{O}_{3}, 99.0 \%\right.$, Sigma Aldrich), Rhodamine 6G (Rh6G) were purchased at Sigma Aldrich, sodium hydroxide (NaOH) (98.0\%, Acros Organics, Fair Lawn, NJ, USA) was purchased at Acros Organics. All the solutions were prepared using Millipore Elix, Burlington, Columbus, NJ, USA $\left(18 \mathrm{M} \Omega \cdot \mathrm{cm}^{2} / \mathrm{cm}\right)$ deionized water.

Sample preparation. Crystalline Rh6G containing M-BA samples were prepared by mixing $20 \mathrm{mM}$ solutions of either melamine or barbituric acid containing similar concentrations of Rh6G dye $\left(0 ; 10^{-7} ; 10^{-6} ; 10^{-5} ; 10^{-4} \mathrm{M}\right)$ cooled to $10^{\circ} \mathrm{C}$ with intensive stirring. Subsequently, the powder precipitation supernatant was decanted and the remaining powdery M-BA was centrifuged and washed three times, until the discoloration of wash waters, and left in deionized water overnight to remove the residual adsorbed dye. Finally, the powder was washed and dried.

The adsorption control experiment was prepared using pure M-BA crystalline powders prepared as described above, without Rh6G presence. The powders were stored in a solution containing $\left(10^{-7} ; 10^{-6} ; 10^{-5} ; 10^{-4} \mathrm{mM}\right)$ for a week and then were rinsed three times and dried.

UV-Vis spectroscopy. All of the spectra were acquired with the Shimadzu UV-1800 UV-spectrophotometer. Prior to all concentration measurements, a series of calibration solutions $(\mathrm{pH}=10)$ of Rh6G was prepared from dry powder and $\mathrm{NaOH}$ solution, a calibration curve (Figure S1) was plotted.

$15 \mathrm{mg}$ of Rh6G containing powders were dissolved in $3 \mathrm{~mL}$ of $\mathrm{NaOH}$ solution (final $\mathrm{pH}=10$ ), and its absorbance was measured against pure $\mathrm{M}-\mathrm{BA}$ solution in $\mathrm{NaOH}$. Concentration was defined from the calibration curve and the final mass of Rh6G in M-BA and its mass fraction was calculated.

Microluminescence spectra for Rh6G solution at different $\mathrm{pH}$ and solid state M-BA and M-BA-Rh6G crystals were acquired using Zeiss Axio Imager. A2m equipped with Ocean Optics QE Pro spectrometer (excitation by HAL 100 and HBO 100).

Scanning electron microscopy and fluorescence microscope images of $M-B A$ crystals with Rh6G. Scanning electron microscopy (SEM) studies were performed with a Hitachi S-3400N scanning electron microscope. Optical photos were acquired at Leica 4500P equipped with halogen UV-source and a red luminescence filter (546 nm excitation, $585 \mathrm{~nm}$ emission).

Powder X-ray diffraction. X-ray diffraction (XRD) measurements of a set of M-BA samples were performed on Shimadzu XRD 6000 with $\mathrm{Cu} \mathrm{K} \alpha$ radiation from diffractionless silicon as a special substrate for the sample with a speed of 1 degree per minute. All calculations were made using regular Shimazu software.

Single crystal X-ray diffraction. Single crystal X-ray diffraction studies were performed at $100 \mathrm{~K}$ using an XtaLAB Synergy-S diffractometer (Rigaku Oxford Diffraction, Oxford, UK) equipped with a hybrid photon counting HyPix-6000 detector that was operated with monochromated, microfocused $\mathrm{CuK} \alpha$ radiation $(\lambda[\mathrm{CuK} \alpha]=1.54184 \AA)$ at $50 \mathrm{kV}$ and $1.0 \mathrm{~mA}$. The data obtained were processed and normalized for polarization, Lorentz, and background effects. The CrysAlisPro [19] program was utilized to perform an empirical absorption correction based on spherical harmonics implemented in the SCALE3 AB- 
SPACK algorithm. The unit-cell parameters (Table S2) were obtained via refining using the least-squares algorithms. The SHELX program $[20,21]$ incorporated in the OLEX2 program package [22] was applied to refine the structure previously solved by a dual-space algorithm. The finalized model incorporated coordinates and anisotropic displacement parameters for all non-hydrogen atoms. The coordinates of carbon-bound hydrogen atoms were found from different Fourier maps and were included in the refinement without any restraints with individual isotropic displacement parameters. Supplementary crystallographic data were uploaded into the Cambridge Crystallographic Data Centre and can be found by quoting the CCDC 2087674 via www.ccdc.cam.ac.uk/structures/ (accessed on 15 May 2021) (see Supplementary Materials).

Quantum chemical computations. The full geometry optimization for all model structures was carried out at the B3LYP-D3/def2-SVP level of theory with the help of the Orca 4.2.1 program package [23]. The RIJCOSX approximation [24,25] utilizing def2SVP/C auxiliary basis set and spin restricted approximation were employed. The convergence tolerances for the geometry optimization were energy change $=5.0 \times 10^{-6} \mathrm{Eh}$, maximal gradient $=3.0 \times 10^{-4} \mathrm{Eh} / \mathrm{Bohr}, \mathrm{RMS}$ gradient $=1.0 \times 10^{-4} \mathrm{Eh} / \mathrm{Bohr}$, maximal displacement $=4.0 \times 10^{-3}$ Bohr, and RMS displacement $=2.0 \times 10^{-3}$ Bohr. The couple perturbed self-consistent field (CPSCF) equations were solved using the conjugated gradient (CG) method with convergence tolerance on a residual of $1.0 \times 10^{-6}$ Eh. The Hessian matrices were calculated for all optimized model structures in order to prove the location of correct stationary points on the potential energy surfaces (no imaginary frequencies were found in all cases) and to estimate the thermodynamic properties (viz. enthalpy, entropy and Gibbs free energy) for all model systems at $298.15 \mathrm{~K}$ and $1 \mathrm{~atm}$. Cartesian atomic coordinates of all model structures are presented in Supplementary Materials.

Molecular dynamics simulation details. Molecular dynamics simulations of M-BA association with Rh6G were performed utilizing the GROMACS program package [26]. Interatomic interactions were described by the OPLS-AA/CM1A force field [27] with topology and charges generated by LigParGen [28-30]. For the water model, the rigid non-polarizable TIP3P [31] model was used. A cut-off for non-bonded and long-range interactions was $1.2 \mathrm{~nm}$. For the calculation of long-range Coulomb interactions, the Particle-Mesh Ewald was used [32]. Energy minimization was performed with the steepest descent algorithm. Simulations were performed in the NPT ensemble at T $=300 \mathrm{~K}$ and $\mathrm{P}=1 \mathrm{~atm}$ in the cubic unit cell (approximate $85 \times 85 \times 85 \mathrm{~A}^{3}$ ). The integration timestep for trajectories' calculations was $2 \mathrm{fs}$, for relaxation $0.5 \mathrm{fs}$. Spatial distribution functions and dimer existence autocorrelation functions were calculated from the resulting trajectories using the TRAVIS program package [33].

\section{Results and Discussion}

\subsection{UV-Vis Spectroscopy of Rh6G Containing M-BA}

Dye containing M-BA particles are explicitly colored with the intensity increasing together with the concentration of Rh6G in the initial solution. To quantitatively estimate the amount of dye encapsulated in a structure, we applied the UV-Vis method (Figure 2a). As expected, the amount of the included dye increases, however, the encapsulation curve does not obey linear dependence with a downward deviation in the high concentration region, which suggests the existence of saturation concentration (Figure 1b). This also correlates with X-ray data, which do not show structural changes or rise in a structural disorder with the increase in impurity content, which also does not support the idea of infinite accommodation of Rh6G in a rigid M-BA structure. The control experiment (Figure S2) showed that adsorption on the M-BA surface does not make a significant contribution to the dye content in the powder. 
a)

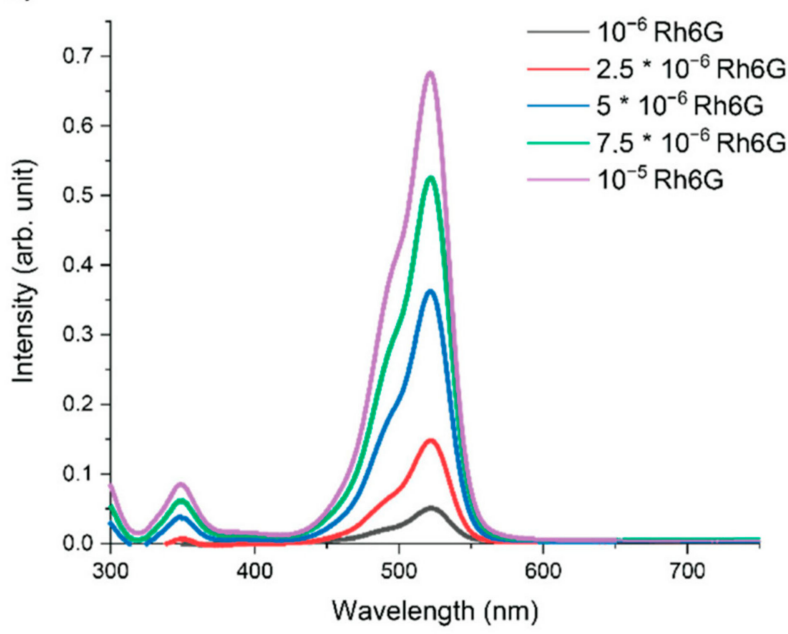

b)

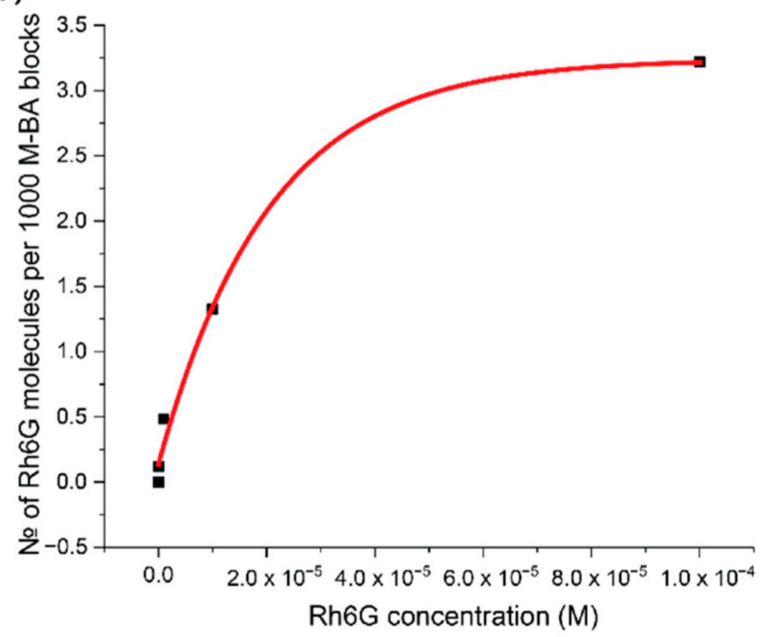

Figure 2. (a) UV-Vis spectra for solution obtained from the M-BA crystals with Rh6G dissolved in $\mathrm{NaOH}$ and (b) the encapsulation curve of Rh6G inside the M-BA crystals.

Quantitative estimation of a fraction of Rh6G is based on the dissolution of a certain mass of M-BA and complete release of incapsulated dye and subsequent calculation of the dye concentration in the solid powder. This approach gives a ratio of a maximum of 3.2 molecules of Rh6G to $1000 \mathrm{M}-\mathrm{BA}$ blocks. It can be represented as the average distance between two Rh6G molecules being no more than 11 M-BA dimers. This estimation shows the surprising stability of the M-BA supramolecular assembly structure.

The comparison of luminescence spectra (Figure S3) of Rh6G solution $\left(C=10^{-5} \mathrm{~mol} / \mathrm{L}\right)$ and different $\mathrm{pH}(1.68 ; 4.01 ; 7.01 ; 9.18 ; 10.01)$ and solid state M-BA-Rh6G reveal that while the spectra of Rh6G at different $\mathrm{pH}$ seem to be the same, the solid state spectra of M-BA-Rh6G have a shift approximately of $15 \mathrm{~nm}$ in a larger wavelength zone. Such a shift can occur due to the stabilizing effect of neighboring barbituric acid and melamine molecules on a LUMO electronic structure and dipole moment of a Rh6G molecule. Luminescence maximum $\mathrm{pH}$ shift measurement of Rh6G solutions indicates that Rh6G-water interactions and Rh6G molecule ionization have a weaker effect on luminescence shift compared with Rh6G-MB interaction.

\subsection{Scanning Electron Microscopy and Fluorescence Microscope Images of M-BA Crystals with Rh6G}

Both methods show the forming of particles in a microscale range, and both show the same crystallographic appearance of the particles. Optical microscopy unambiguously demonstrates that Rh6G is included in the particles, dying them into pink color; the color varies from white to intensive pink with the rise of concentration of Rh6G in reaction media. Moreover, the prolonged washing of the particles in the deionized water utilizing ultrasonication did not lead to color change which indicates that Rh6G moiety is absorbed in the structure and not adsorbed onto the surface of the particles. Both Figures 3 and 4 show an obvious tendency in simplification of the crystalline particles' shape toward single crystals in the reaction media containing the highest Rh6G concentration. At the same time, a growth in intensity of luminescence in the characteristic rhodamine channel (excitation $546 \mathrm{~nm}$, emission $585 \mathrm{~nm}$ ) is observed. Scanning electron microscopy shows the tendency of surface quality raising with the increase in Rh6G concentration. The particle size remains almost the same and thus is not affected by Rh6G inclusion. Figure 1 shows fluorescence microscopy images of melamine-barbiturate crystals with and without the inclusion of Rh6G. The Rh6G presence in the reaction medium leads to BA-Rh6G soluble clusters formation and therefore leading to deficiency of one of the components of the reaction. Such deficiency, in turn, leads to a decrease in nuclei formation rate and crystal growth speed, which influence the resulting shape of crystals. 

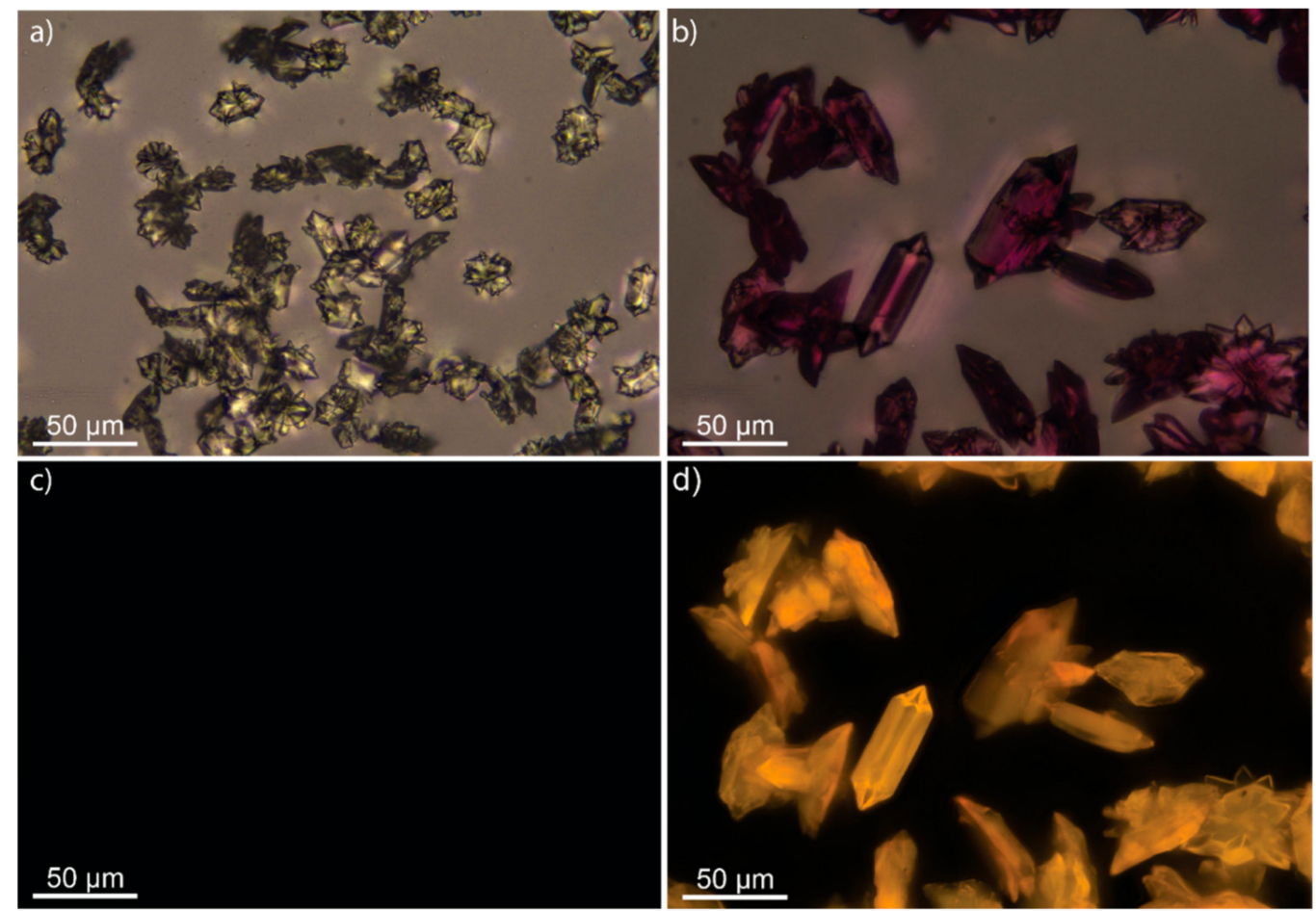

Figure 3. Crystals of M-BA (a) and M-BA with Rh6G (b) in transmitted light and particle luminescence of pure M-BA (c) and M-BA with Rh6G (d).
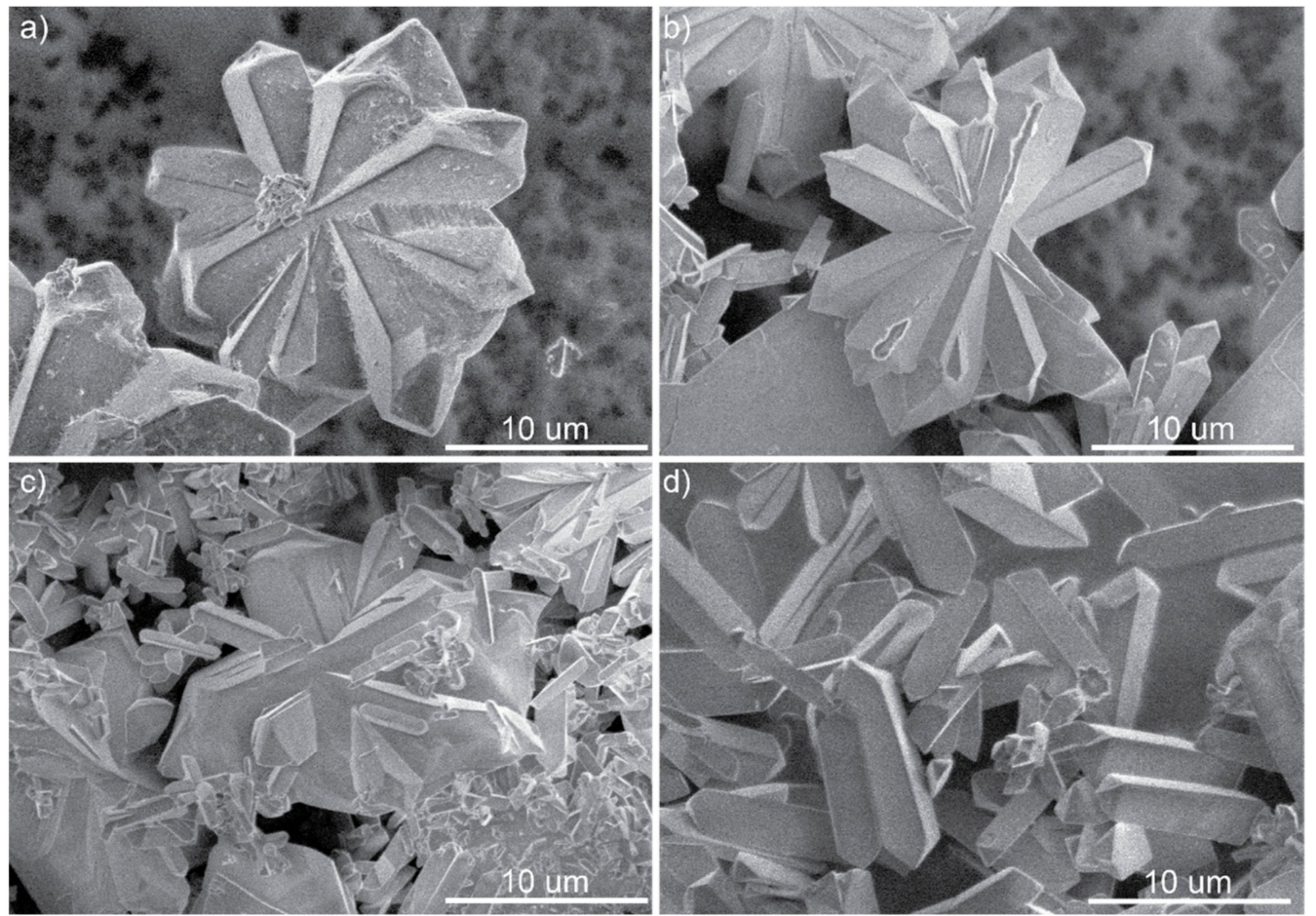

Figure 4. SEM images of M-BA particles with various concentrations of Rh6G: $0 \mathrm{M}(\mathbf{a}), 10^{-7} \mathrm{M}(\mathbf{b}), 10^{-6} \mathrm{M}$ (c), $10^{-4} \mathrm{M}(\mathrm{d})$.

\subsection{Powder X-ray Diffraction}

In order to get insight into the crystallinity of the resulting samples and the influence of Rh6G on structure type and quality, powder X-ray diffraction was utilized. The resulting XRD patterns (Figure 5) perfectly match the pattern for pure melamine barbiturate with no excess peak emergency or intensity changes. Moreover, the inclusion of Rh6G molecules inside the crystals does not strongly affect their crystallinity (Table S1) regardless of the 
number of dye molecules in the lattice. This effect may be related to the fact that Rh6G has a large aromatic ring that can form the $\pi-\pi$ stacking with the barbituric acid ring, which leads to the Rh6G being locally injected into the crystalline lattice in place of the corresponding M-BA layer. To get a more thorough look into the processes occurring on a molecular level, we utilized computational methods such as DFT and Classical Molecular Dynamics simulations.

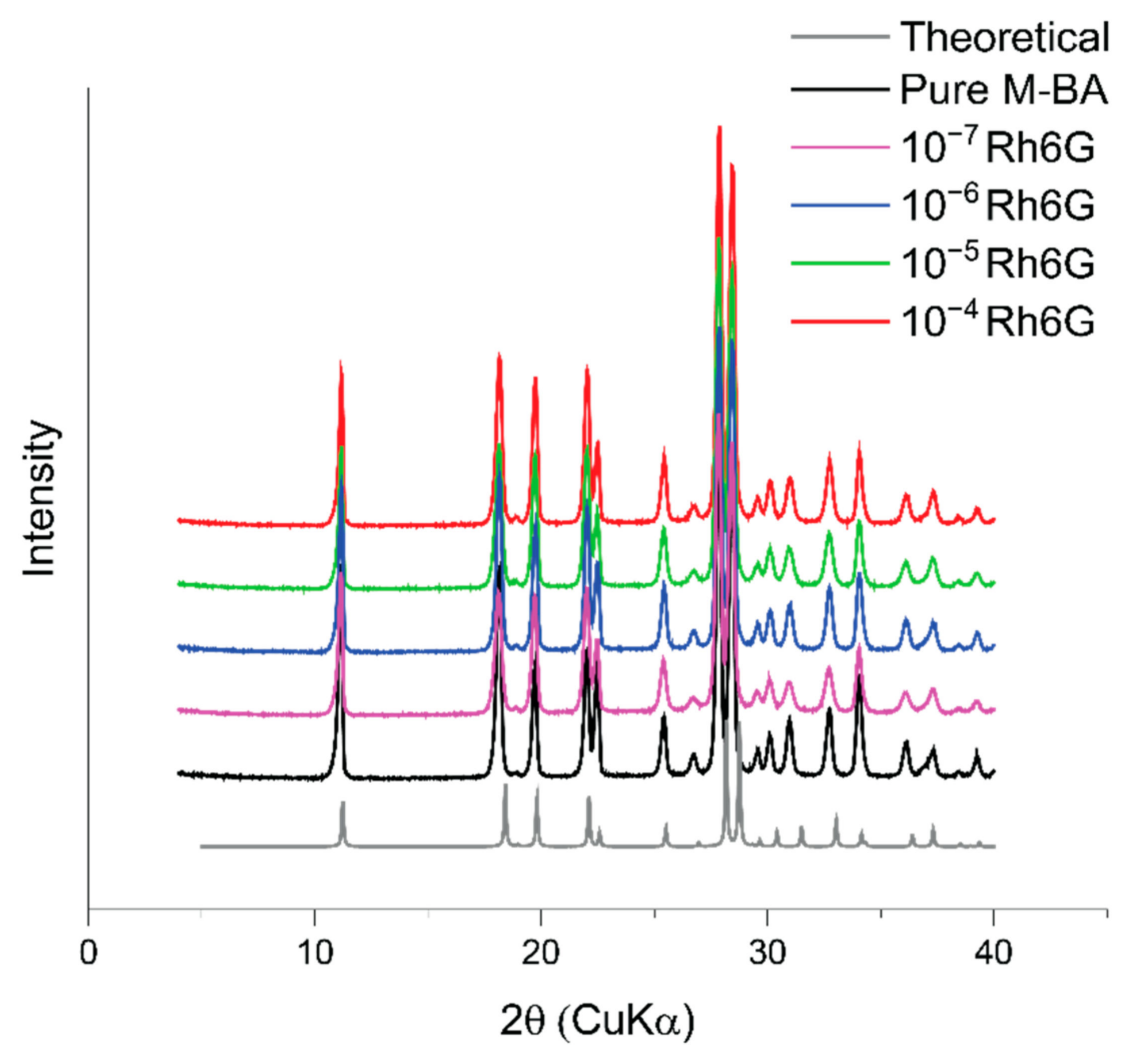

Figure 5. Experimental and theoretical XRD patterns for M-BA crystals with different concentrations of Rh6G. The theoretical pattern (grey) is for pure M-BA crystal.

\subsection{Single Crystal X-ray Diffraction}

Two cif files related to the M-BA can be found in the CCDC database under the $1,185,866$ and 1,185,867 deposition numbers. However, the first one does not contain atomic coordinates, has similar unit cell and refinement parameters to the latter file and refers to a paper [34] that describes the structure of another $\mathrm{N}, \mathrm{N}^{\prime}$-diphenylmelamine.5,5diethylbarbituric acid complex. Thus, it should be pointed that the crystal structure of M-BA was first described in the latter paper of the same scientific group [35] and the first file appears in the database by mistake. Although the structure of M-BA have been reported previously [35], and our study confirms that structural model, the use of good-quality crystals and modern XRD equipment made it possible to refine the structure of M-BA with higher precision.

There are one melamine and one barbituric acid crystallographically unique molecules in the structure of M-BA. Both molecules arrange special sites on the 2-fold axes, which pass through $\mathrm{N} 1-\mathrm{C} 1-\mathrm{N} 4$ and $\mathrm{O} 1-\mathrm{C} 3-\mathrm{C} 5$ atoms, thus having half of the atoms as equivalents (Figure 6a). Molecules of melamine and barbituric acid in the structure of M-BA are linked through the system of strong $\mathrm{N}-\mathrm{H} \cdots \mathrm{N}$ and $\mathrm{N}-\mathrm{H} \cdots \mathrm{O}$ hydrogen bonds (Figure 6a), thus forming infinite flat chains. These chains in turn are arranged parallel to each other, forming slabs parallel to the (001). There are two types of such slabs in the unit cell of M-BA, in which planes of infinite chains, and of melamine and barbituric acid molecules themselves, are arranged parallel to (120) and (1-20) with the twist angle between the planes of chains 
equal to $80.1(2)^{\circ}$ (Figure $6 \mathrm{~b}$ ). Such a type of molecular arrangement is not unique and was recently reported, e.g., in [11]. But in contrast to the arrangement of uric acid molecules [36], melamine/barbituric acid complexes do not have additional $\pi$-stacking interaction between the flat chains within the slab. Although the normal distance between the mean planes of chains is rather short (3.210(4) $\AA$ ) and comparative to that in [36] (3.241(3) - 3.311(8) $\AA$ ), chains in the structure of M-Ba are displaced relative to each other in such a way that the cyclic fragment of one chain in normal projection is arranged opposite the H-bonding system between melamine and barbituric acid molecules in the adjacent chain.
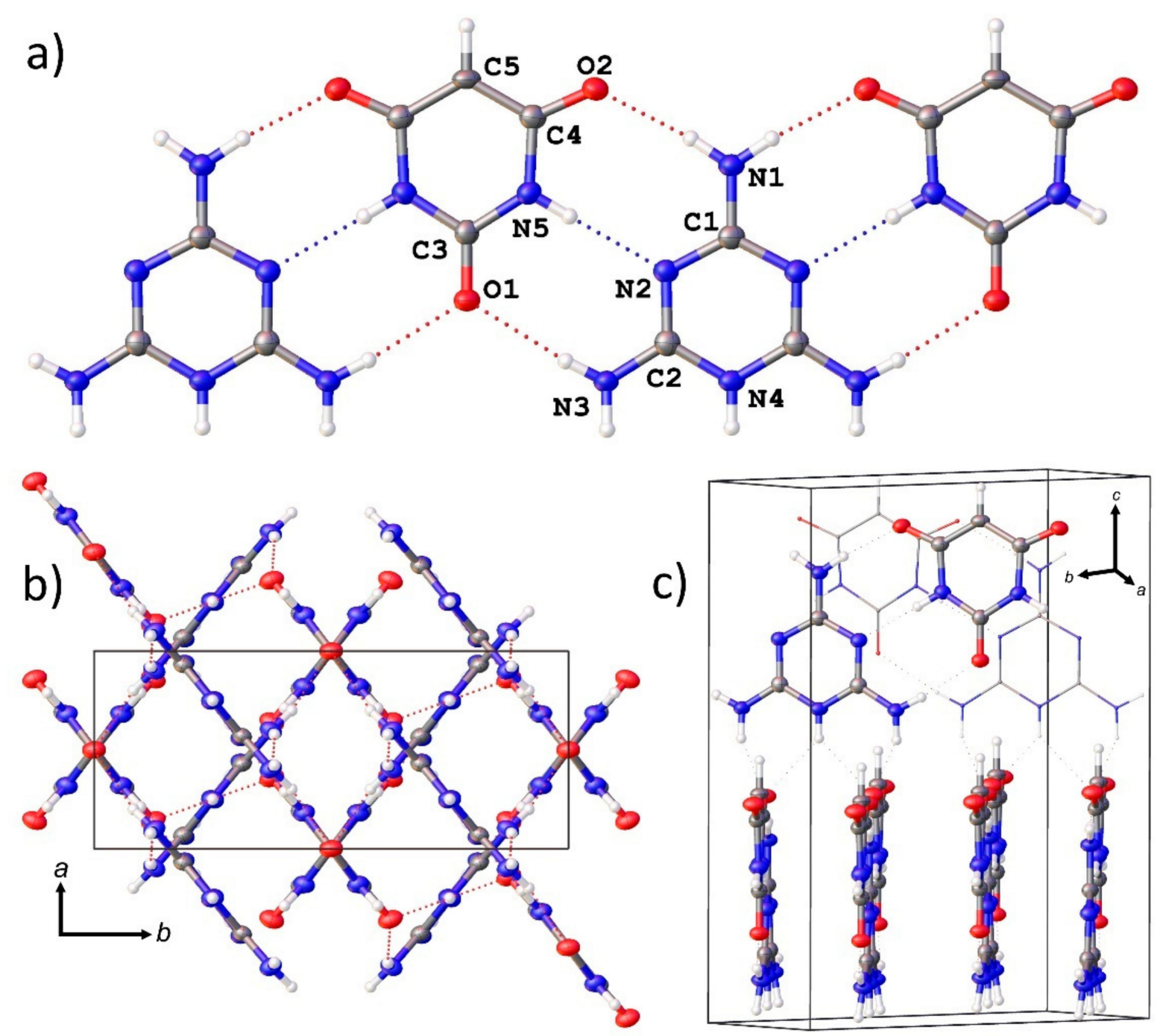

Figure 6. An arrangement of the melamine and barbituric acid molecules in a flat infinite chain in the structure of M-BA (a); only crystallographically non-equivalent fragments are labeled. Crystal structure of M-BA, projections along the [001] (b) and approximately along the [210] (c) directions. Legend: C, N, O and H atoms are shown as grey, blue, red and white spheres, respectively. Thermal ellipsoids are drawn at the $50 \%$ probability level. Molecules drawn in a capped-stick style in (c) correspond to the second chain. Red and blue dotted line $=\mathrm{H}$ bonds.

\subsection{Quantum Chemistry Calculations}

We have investigated the Gibbs free energy of association for small supramolecular complexes with the inclusion of Rh6G. Figure 7 shows the trend of descending energy with an increasing number of M-BA molecules in the supramolecular structure. While M-BA dimer seems to be slightly more energetically favorable than the dimers with the Rh6G, the supramolecular structures with more molecules favor the inclusion of Rh6G. This indicates that the formation of the structures with long-range order is more beneficial energetically 
with the inclusion of Rh6G molecules. Moreover, it seems that Rh6G tends to bind to BA molecules in the supramolecular structure. Analysis of Rh6G-BA and Rh6G-M dimers shows that the most energetically preferable group for binding of $\mathrm{M}$ and $\mathrm{BA}$ molecules is an ester group of Rh6G. In this case, the M and BA molecules bind in such a way that they not only form a hydrogen bond with the ester group, but also achieve $\pi-\pi$ stacking with the large aromatic plane of Rh6G. Moreover, a quick analysis of optimized geometries indicates that the most stable M-BA associates form with the largest amount of hydrogen bonds possible for the given structure.

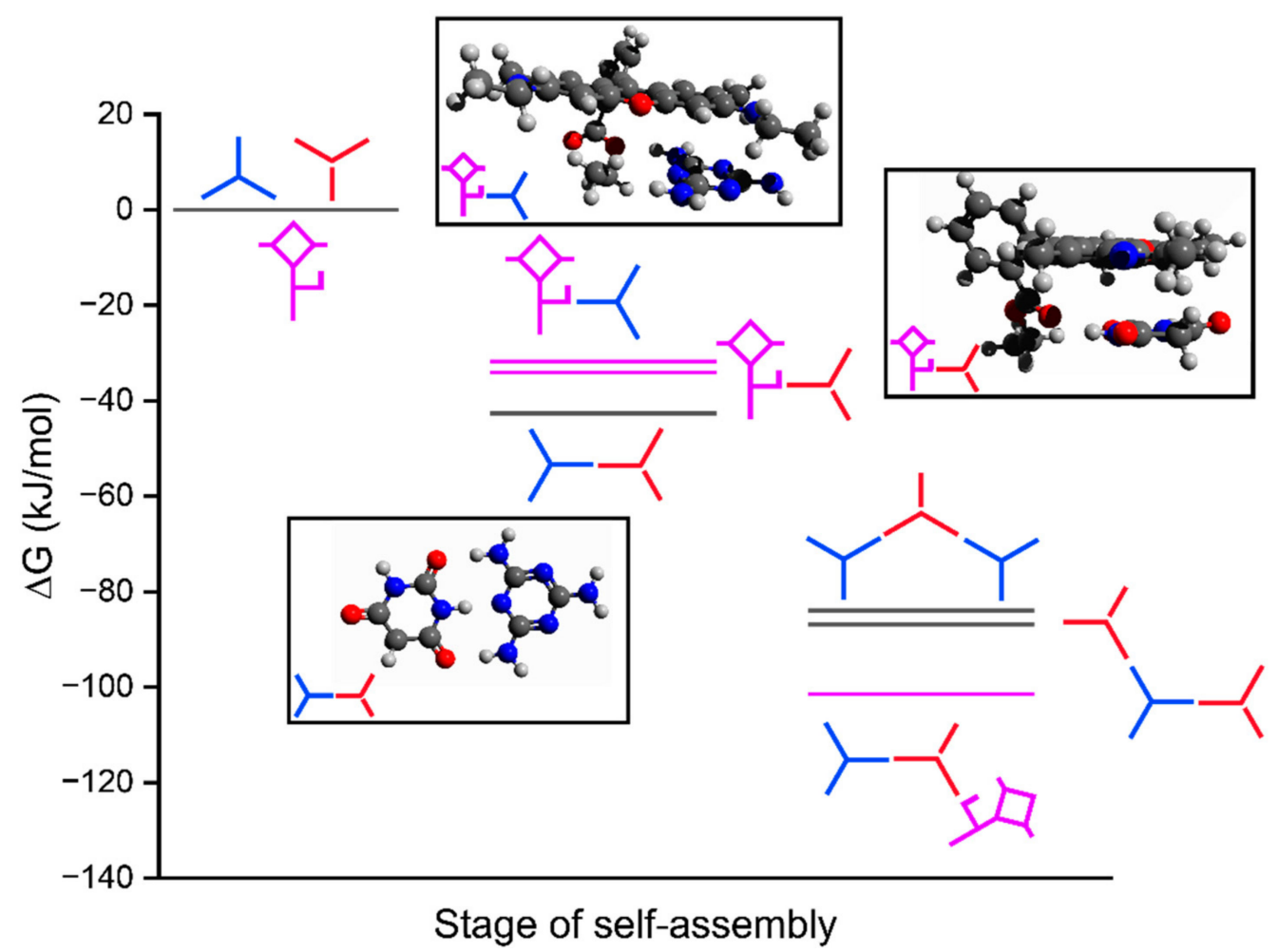<smiles>C#CC1CC(C)CC1C</smiles>

Figure 7. Change of Gibbs free energy of association during the formation of M-BA supramolecular assemblies with Rh6G with respect to initial components.

\subsection{Molecular Dynamics Simulations}

Four molecular dynamics simulations were performed utilizing the GROMACS program package: two in the presence of Rh6G molecules and two without these additives. While the DFT analysis provides information about the optimized geometries and stability of small associates in the "idealized" gaseous phase, the Molecular Dynamics simulations expand this information with data on the formation of nuclei and Rh6G integration in the structure of M-BA associates. There were two types of simulation boxes: with and without the Rod6G molecule. The boxes contained 19,000 water molecules, $40 \mathrm{M}, 40 \mathrm{BA}$ and 0 or 1 Rh6G. Initially, all molecules were deposited randomly in the unit cell. After that, the energy minimization and quick relaxation at small integration timestep ( $0.5 \mathrm{fs})$ for $50 \mathrm{ps}$ were performed. Afterwards, the trajectory was calculated with NPT ensemble at T $=300 \mathrm{~K}$ and $\mathrm{P}=1 \mathrm{~atm}$ for $200 \mathrm{~ns}$. 
The data in Figure 8 represent the time-dependent radial distribution functions (RDFs) between centers of mass of M and BA molecules of systems with (Figure 8a) and without (Figure $8 b$ ) inclusion of Rh6G molecule. The comparison of the two maps shows that, in both cases, the stable nuclei did not form within $200 \mathrm{~ns}$ of simulation, although the formation of a quasi-stable cluster was observed. It seems that the time needed to form the quasi-stable nuclei is larger in the presence of Rh6G molecules. The first occurrence of quasi-stable cluster happens at the $15 \mathrm{~ns}$ for the system without Rh6G and at the $65 \mathrm{~ns}$ for the system with Rh6G.

a)

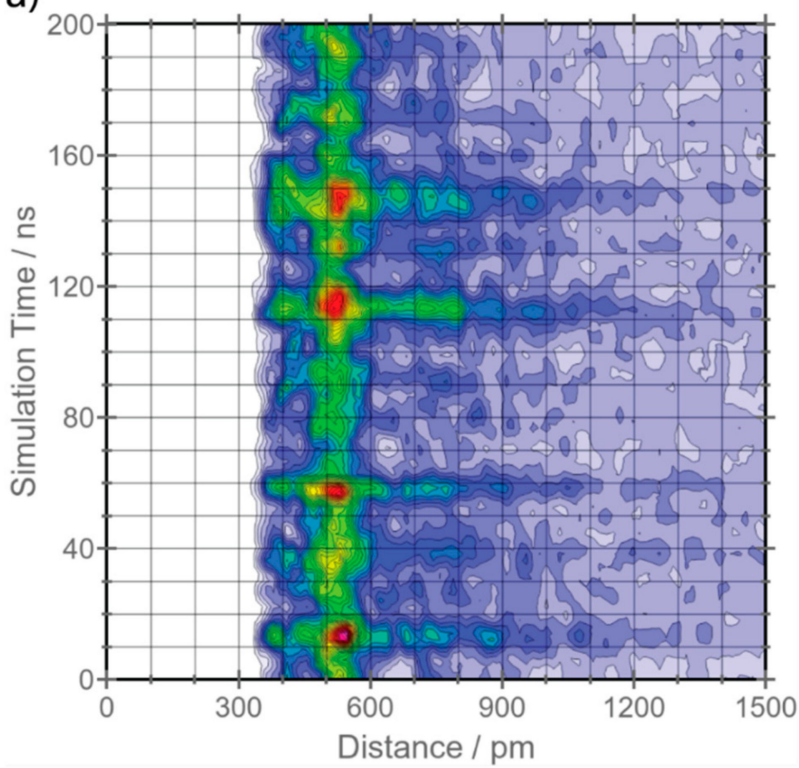

b)

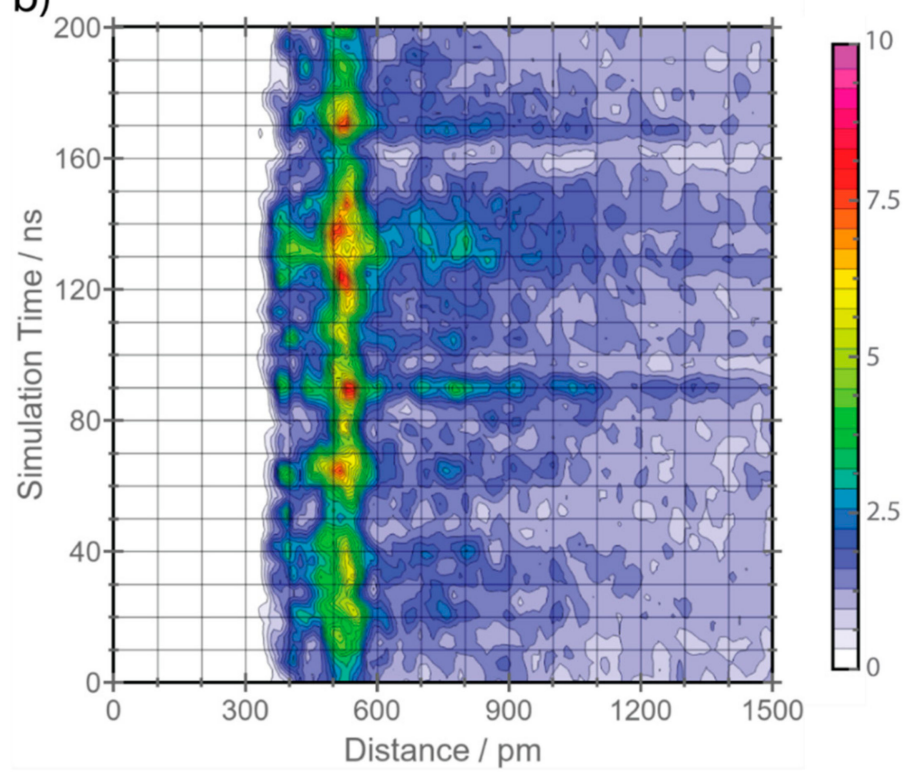

Figure 8. Time-dependent radial distribution function between centers of mass of M and BA molecules for systems without (a) and with (b) Rh6G.

Figure 9 shows the combined data obtained from the resulting trajectories. Spatial distribution functions (SDFs) of BA and M around Rh6G are shown in Figure 9c,d, respectively. The BA and $\mathrm{M}$ molecules both form a $\pi-\pi$ stacking with the largest plane of Rh6G. Moreover, the BA molecules seem to be associated with the ester group of Rh6G. Figure $9 \mathrm{~h}$ shows the dimer existence autocorrelation function for the following dimers: M-BA; Rh6G-M and Rh6G-BA. This figure shows the average lifetime of the heterogeneous dimers in the simulation. It is clearly seen that the lifetime of these dimers is quite low (around $1 \mathrm{~ns}$ ), although the dimers with Rh6G have quite a larger lifetime than M-BA dimers. Figure 9i-k shows the number of neighbors' occurrence throughout the simulation for the dimers Rh6G-M, Rh6G-BA and M-BA, respectively. It shows that Rh6G has more BA than $\mathrm{M}$ neighbors. The radial distribution functions for all possible pairs of molecules (except Rh6G molecule because there is only one in the simulation box) are represented in Figure $9 \mathrm{~g}$. The RDFs between centers of mass of molecules (Figure 9g), spatial distribution functions of $\mathrm{M}$ around $\mathrm{M}$ (Figure 9f) and BA around BA (Figure 9a) show that homogeneous dimers tend to form very consistently with BA-BA dimers forming $\pi-\pi$ stacking and $\mathrm{M}-\mathrm{M}$ forming planar dimers. The SDFs of M-BA dimer-the $\mathrm{M}$ around $\mathrm{BA}$ and BA around $\mathrm{M}$-indicate that predominantly the M-BA dimer forming is achieved through planar and $\pi-\pi$ stacking. Moreover, the planar stacking occurs in the direction of the formation of the largest numbers of hydrogen bonds, which is consistent with DFT data. 
a)
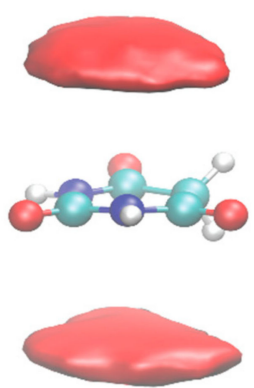

b)

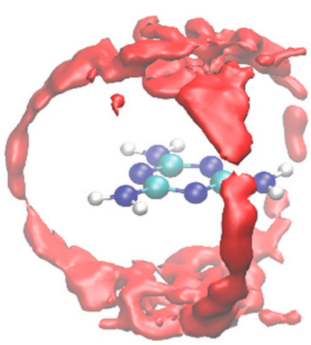

h)

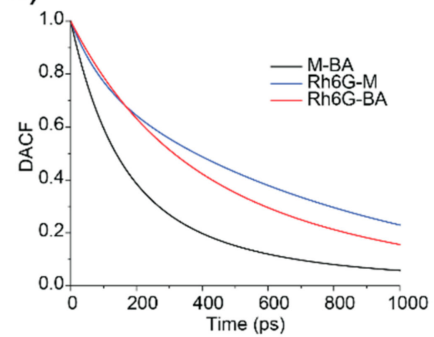

c)

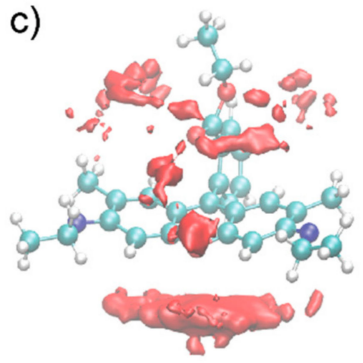

d)

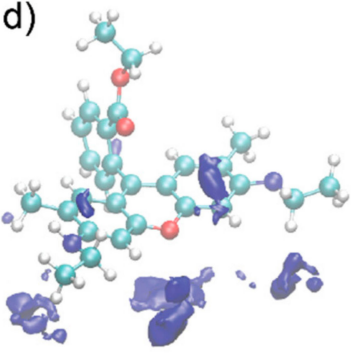

g)

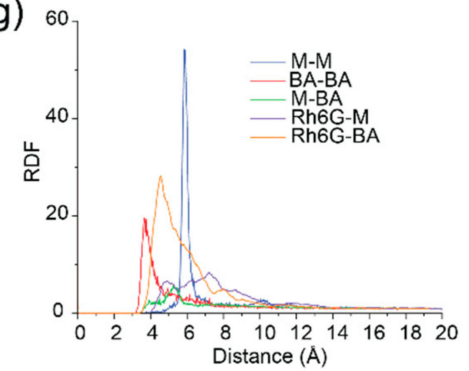

i)

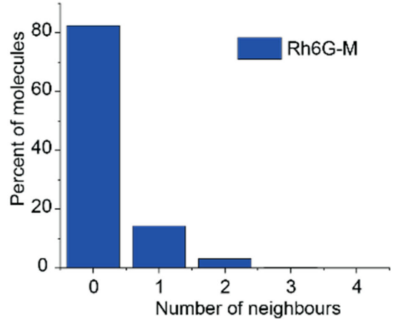

j)

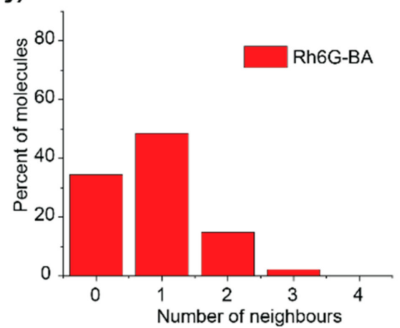

e)

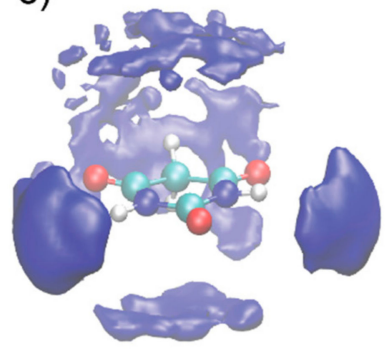

f)

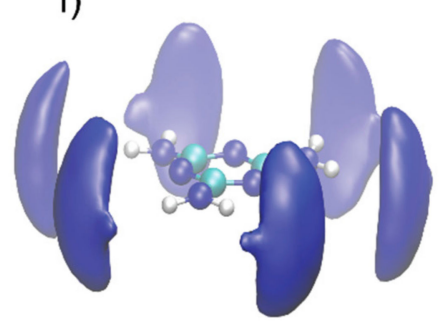

k)

Figure 9. Spatial distribution functions for BA around (a) BA, (b) MEL and (c) Rh6G in the water solution; spatial distribution functions for MEL around (d) Rh6G, (e) BA and (f) MEL in the water solution (g) radial distribution functions between centers of mass of M-M, BA-BA, M-BA, Rh6G-M and Rh6G-BA (h) dimer existence autocorrelation functions for M-BA, Rh6G-M and Rh6G-BA (i-k) number of neighbors Rh6G-M, Rh6G-BA and M-BA, respectively.

\section{Conclusions}

In this study, we used the melamine barbiturate supramolecular system as a host for encapsulating Rh6G molecules. SEM and fluorescent microscopy images of melamine barbiturate crystals with and without Rh6G show clear inclusion of dye molecules inside the organic crystals. Moreover, the presence of Rh6G drastically improves the symmetry of melamine barbiturate crystals. pXRD data suggest that there is no rise in structural disorder with the increase in Rh6G concentration inside the crystals. The shape of the encapsulation curve indicates the existence of saturation concentration-a maximum of 3.2 Rh6G molecules per 1000 M-BA blocks. Quantum chemical calculations show the energetical preferability of supramolecular assembly of M-BA with Rh6G. Moreover, it seems that BA and $\mathrm{M}$ tend to form $\pi-\pi$ stacking with a large aromatic ring of Rh6G, which is consistent with Molecular Dynamics data. Classical Molecular Dynamics simulations indicate the overall tendency in Rh6G binding BA (and to a lesser degree M) molecules. Such a binding forms the deficiency of one reagent for crystallization and therefore leads to a deterioration in the nucleation and crystallization rate promoting the growth of larger and more symmetrical organic crystals. Hence, we conclude that not only melamine barbiturate supramolecular assembly is capable of effectively encapsulating Rh6G, but the inclusion of such dye molecules leads to a drastic improvement in the shape of resulting organic crystals. 
Supplementary Materials: The following are available online at https:/ / www.mdpi.com/article/10 .3390/sym13071119/s1, Figure S1: Calibration curve for M-BA crystals dissolved in NaOH solution; Figure S2: UV-Vis spectra for solution obtained from dissolved M-BA crystals with (red curve) and without Rh6G inside the crystal. Prior to the dissolution, the crystals were placed inside Rh6G solution with given concentrations: 10-7, 10-6 and 10-5 (black, blue and red curves, respectively); after that, they were washed in distilled water; Figure S3: Luminescence spectra of Rh6G solutions at different $\mathrm{pH}$ and luminescence spectra of solid state M-BA and M-BA-Rh6G; Table S1: Crystallinity data for M-BA and M-BA-Rh6G crystals; Table S2: Crystallographic data for M-BA.

Author Contributions: Conceptualization, E.V.S. and P.V.N.; methodology, V.V.S.; analysis, E.V.B. and V.V.G.; computation P.V.N., A.S.N., N.D.K. and N.D.O.; investigation, A.D.S., V.V.G. and A.A.T.; writing-original draft preparation, P.V.N. and V.V.S.; writing-review and editing, E.V.S., A.S.N., N.D.K. and N.D.O.; visualization, P.V.N.; supervision, E.V.S.; project administration, E.V.S.; funding acquisition, E.V.S. All authors have read and agreed to the published version of the manuscript.

Funding: This research was funded by the Ministry of Science and Higher Education of Russian Federation, goszadanie no. 2019-1075.

Data Availability Statement: The data presented in this study are available in Supplementary Materials of this paper.

Acknowledgments: The SCXRD studies were performed at the X-ray Diffraction Centre of the St. Petersburg State University. SEM studies were performed at "Geomodel". Luminescence studies were performed at ITMO center of collective use of scientific equipment. ITMO Fellowship and Professorship Program is acknowledged for infrastructural support.

Conflicts of Interest: The authors declare no conflict of interest.

\section{References}

1. Matos, J.C.; Pereira, L.C.J.; Waerenborgh, J.C.; Gonçalves, M.C. Encapsulation of Active Molecules in Pharmaceutical Sector: The Role of Ceramic Nanocarriers; Elsevier: Amsterdam, The Netherlands, 2020; ISBN 9780128193631.

2. Ye, C.; Chi, H. A review of recent progress in drug and protein encapsulation: Approaches, applications and challenges. Mater. Sci. Eng. C 2018, 83, 233-246. [CrossRef]

3. Jiwpanich, S.; Ryu, J.H.; Bickerton, S.; Thayumanavan, S. Noncovalent encapsulation stabilities in supramolecular nanoassemblies. J. Am. Chem. Soc. 2010, 132, 10683-10685. [CrossRef]

4. Arunkumar, E.; Forbes, C.C.; Smith, B.D. Improving the properties of organic dyes by molecular encapsulation. Eur. J. Org. Chem. 2005, 4051-4059. [CrossRef]

5. Liu, C.; Gao, C.; Yan, D. Synergistic supramolecular encapsulation of amphiphilic hyperbranched polymer to dyes. Macromolecules 2006, 39, 8102-8111. [CrossRef]

6. Müller, M.; Zentel, R.; Maka, T.; Romanov, S.G.; Sotomayor Torres, C.M. Dye-containing polymer beads as photonic crystals. Chem. Mater. 2000, 12, 2508-2512. [CrossRef]

7. Furumi, S. Self-assembled organic and polymer photonic crystals for laser applications. Polym. J. 2013, 45, 579-593. [CrossRef]

8. Sola-Llano, R.; Gartzia-Rivero, L.; Oliden-Sanchez, A.; Bañuelos, J.; Arbeloa, I.L.; Martínez-Martínez, V. Dye encapsulation into one-dimensional zeolitic materials for optical applications. Chem. Silica Zeolite Based Mater 2019, 229-248. [CrossRef]

9. Ibrahim, M.S.; Etaiw, S.E.D.H. Supramolecular host-guest systems as frameworks for excitation energy transfer. Spectrochim. Acta Part A Mol. Biomol. Spectrosc. 2002, 58, 373-378. [CrossRef]

10. Ramamurthy, V.; Jockusch, S.; Porel, M. Supramolecular photochemistry in solution and on surfaces: Encapsulation and dynamics of guest molecules and communication between encapsulated and free molecules. Langmuir 2015, 31, 5554-5570. [CrossRef]

11. Sun, J.K.; Sobolev, Y.I.; Zhang, W.; Zhuang, Q.; Grzybowski, B.A. Enhancing crystal growth using polyelectrolyte solutions and shear flow. Nature 2020, 579, 73-79. [CrossRef]

12. Björk, J.; Hanke, F.; Palma, C.A.; Samori, P.; Cecchini, M.; Persson, M. Adsorption of aromatic and anti-aromatic systems on graphene through $\pi-\pi$ Stacking. J. Phys. Chem. Lett. 2010, 1, 3407-3412. [CrossRef]

13. Dobberschütz, S.; Nielsen, M.R.; Sand, K.K.; Civioc, R.; Bovet, N.; Stipp, S.L.S.; Andersson, M.P. The mechanisms of crystal growth inhibition by organic and inorganic inhibitors. Nat. Commun. 2018, 9, 1-6. [CrossRef]

14. Shilovskikh, V.V.; Timralieva, A.A.; Nesterov, P.V.; Novikov, A.S.; Sitnikov, P.A.; Konstantinova, E.A.; Kokorin, A.I.; Skorb, E.V. Melamine-barbiturate supramolecular assembly as a pH-dependent organic radical trap material. Chem. A Eur. J. 2020, 26, 16603-16610. [CrossRef] [PubMed]

15. Orekhov, N.; Kondratyuk, N.; Logunov, M.; Timralieva, A.; Shilovskikh, V.; Skorb, E.V. Insights into the early stages of melamine cyanurate nucleation from aqueous solution. Cryst. Growth Des. 2021, 21, 1984-1992. [CrossRef]

16. Shilovskikh, V.V.; Timralieva, A.A.; Belogub, E.V.; Konstantinova, E.A.; Kokorin, A.I.; Skorb, E.V. Radical activity of binary melamine-based hydrogen-bonded self-assemblies. Appl. Magn. Reson. 2020, 51, 939-949. [CrossRef] 
17. Mane, S.R.; Sathyan, A.; Shunmugam, R. Barbiturate derived amphiphilic homopolymers: Synthesis, characterization, selfassembly and anticancer drug delivery. Ther. Deliv. 2019, 10, 419-431. [CrossRef] [PubMed]

18. Roy, B.; Bairi, P.; Nandi, A.K. Supramolecular assembly of melamine and its derivatives: Nanostructures to functional materials. RSC Adv. 2014, 4, 1708-1734. [CrossRef]

19. Rigaku Corporation. Crys AlisPro Software System, Version 1.171.40.50a; Rigaku Oxford Diffraction: Oxford, UK, 2019.

20. Sheldrick, G.M. SHELXT-Integrated space-group and crystal structure determination. Acta Cryst. 2015, A71, 3-8. [CrossRef]

21. Sheldrick, G.M. Crystal structure refinement with SHELXL. Acta Cryst. 2015, C71, 3-8. [CrossRef]

22. Dolomanov, O.V.; Bourhis, L.J.; Gildea, R.J.; Howard, J.A.K.; Puschmann, H. OLEX2: A complete structure solution, refinement and analysis program. J. Appl. Cryst. 2009, 42, 339-341. [CrossRef]

23. Neese, F. The ORCA program system. Wiley Interdiscip. Rev. Comput. Mol. Sci. 2012, 2, 73-78. [CrossRef]

24. Neese, F.; Wennmohs, F.; Hansen, A.; Becker, U. Efficient, approximate and parallel Hartree-Fock and hybrid DFT calculations. A "chain-of-spheres" algorithm for the Hartree-Fock exchange. Chem. Phys. 2009, 356, 98-109. [CrossRef]

25. Neese, F. An Improvement of the resolution of the identity approximation for the formation of the coulomb matrix. J. Comput. Chem. 2003, 24, 1740-1747. [CrossRef] [PubMed]

26. Berendsen, H.J.C.; van der Spoel, D.; van Drunen, R. GROMACS: A message-passing parallel molecular dynamics implementation. Comput. Phys. Commun. 1995, 91, 43-56. [CrossRef]

27. Jorgensen, W.L.; Maxwell, D.S.; Tirado-Rives, J. Development and testing of the OPLS all-atom force field on conformational energetics and properties of organic liquids. J. Am. Chem. Soc. 1996, 118, 11225-11236. [CrossRef]

28. Jorgensen, W.L.; Tirado-Rives, J. Potential energy functions for atomic-level simulations of water and organic and biomolecular systems. Proc. Natl. Acad. Sci. USA 2005, 102, 6665-6670. [CrossRef]

29. Dodda, L.S.; Vilseck, J.Z.; Tirado-Rives, J.; Jorgensen, W.L. 1.14*CM1A-LBCC: Localized bond-charge corrected CM1A charges for condensed-phase simulations. J. Phys. Chem. B 2017, 121, 3864-3870. [CrossRef]

30. Dodda, L.S.; De Vaca, I.C.; Tirado-Rives, J.; Jorgensen, W.L. LigParGen web server: An automatic OPLS-AA parameter generator for organic ligands. Nucleic Acids Res. 2017, 45, W331-W336. [CrossRef]

31. Jorgensen, W.L.; Chandrasekhar, J.; Madura, J.D.; Impey, R.W.; Klein, M.L. Comparison of simple potential functions for simulating liquid water. J. Chem. Phys. 1983, 79, 926-935. [CrossRef]

32. Essmann, U.; Perera, L.; Berkowitz, M.L.; Darden, T.; Lee, H.; Pedersen, L.G. A smooth particle mesh Ewald method. J. Chem. Phys. 1995, 103, 8577-8593. [CrossRef]

33. Brehm, M.; Kirchner, B. TRAVIS-A free analyzer and visualizer for monte carlo and molecular dynamics trajectories. J. Chem. Inf. Model. 2011, 51, 2007-2023. [CrossRef] [PubMed]

34. Zerkowski, J.A.; Seto, C.T.; Wierda, D.A.; Whitesides, G.M. The design of organic structures in the solid state: Hydrogen-bonded molecular "tapes". J. Am. Chem. Soc. 1990, 112, 9025-9026. [CrossRef]

35. Zerkowski, J.A.; MacDonald, J.C.; Whitesides, G.M. Investigations into the robustness of secondary and tertiary architecture of hydrogen-bonded crystalline tapes. Chem. Mater. 1994, 6, 1250-1257. [CrossRef]

36. Izatulina, A.R.; Gurzhiy, V.V.; Krzhizhanovskaya, M.G.; Chukanov, N.V.; Panikorovskii, T.L. Thermal behavior and phase transition of uric acid and its dihydrate form, the common biominerals uricite and tinnunculite. Minerals 2019, 9, 373. [CrossRef] 